\title{
Long-Range Axonal Calcium Sweep Induces Axon Retraction
}

\author{
Ryuji X. Yamada, ${ }^{1}$ Takuya Sasaki, ${ }^{1}$ Junya Ichikawa, ${ }^{1}$ Ryuta Koyama, ${ }^{1}$ Norio Matsuki, ${ }^{1}$ and Yuji Ikegaya ${ }^{1,2}$ \\ ${ }^{1}$ Laboratory of Chemical Pharmacology, Graduate School of Pharmaceutical Sciences, The University of Tokyo, Tokyo 113-0033, Japan, and ${ }^{2}$ Precursory \\ Research for Embryonic Science and Technology, Japan Science and Technology Agency, Tokyo 102-8666, Japan
}

\begin{abstract}
Axon guidance molecules trigger a cascade of local signal in growth cones and evoke various morphologic responses, including axon attraction, repulsion, elongation, and retraction. However, little is known about whether subcellular compartments, other than axonal growth cones, control axon outgrowth. We found that in isolated dentate granule cells, local application of glutamate to the somatodendritic areas, but not the axon itself, induced rapid axon retraction, during which a calcium wave propagated from the somata to the axon terminals. The calcium wave and axon retraction were both inhibited by blockade of voltage-sensitive calcium channels and intracellular calcium dynamics. A combination of perisomatic application of calcium ionophore and depolarizing current injection induced axonal calcium sweep and axon retraction. Thus, perisomatic environments can modulate axon behavior through long-range intracellular communication.
\end{abstract}

Key words: axon; axon guidance; calcium; culture; dentate gyrus; glutamate; glutamate receptor; granule cell; hippocampus

\section{Introduction}

During brain development, immature neurons project axons over long distances to target cells. In this process, a key player is a structural expansion present at the tip of a growing axon (i.e., a growth cone). The growth cone is highly motile and actively turns, advances, and retreats in response to extracellular guidance cues. The growth cone converts these local cues to an intracellular signal cascade that determine the direction and speed of axon growth (Tessier-Lavigne and Goodman, 1996; Huber et al., 2003). In Xenopus spinal neurons and retinal neurons, axons preserve chemotactic behaviors even when surgically transected from the cell bodies (Campbell and Holt, 2001; Ming et al., 2002). Thus, neurite outgrowth seems to be totally controlled by local environments surrounding the growth cones.

Granule cells, the principal excitatory neurons in the dentate gyrus of the hippocampal formation, extend their axons into the hilus and CA3 stratum lucidum to make synapses with target hilar/CA3 neurons. We previously established an experimental system to cultivate isolated granule cells (Yamada et al., 2005). Using this system, we disclosed that bath application of glutamate collapses growth cones of dendrites as well as axons. Glutamate is therefore likely to play an important role in network formation of dentate granule cells. In the previous work, however, glutamate was uniformly applied to the entire neurons, and its local effect on growth cones was not determined. In the present study, we

Received Jan. 3, 2008; revised March 13, 2008; accepted March 19, 2008.

This work was supported in part by a Grant-in-Aid for Science Research on Priority Areas (Elucidation of Neural Network Function in the Brain: KAKENHI 18021008, 17023015), a Grant-in-Aid for Science Research (KAKENHI 17650090, 17689004, 19659013), The Research Foundation for Pharmaceutical Sciences, Takeda Science Foundation, and The Asahi Glass Foundation. We thank Dr. Kenneth D. Harris for his critical comments on this manuscript

Correspondence should be addressed to Yuji Ikegaya, Laboratory of Chemical Pharmacology, Graduate School of Pharmaceutical Sciences, The University of Tokyo, 7-3-1 Hongo, Bunkyo-ku, Tokyo 113-0033, Japan. E-mail: ikegaya@mol.f.u-tokyo.ac.jp.

DOI:10.1523/JNEUROSCI.0019-08.2008

Copyright $\odot 2008$ Society for Neuroscience $\quad 0270-6474 / 08 / 284613-06 \$ 15.00 / 0$ introduced a local drug-perfusion system to examine how local glutamate affects the behavior of axon growth cones. As a result, we unexpectedly found that glutamate did not alter the growth cone dynamics when applied directly to the axons, but it readily changed the axon behavior when applied to a somatodendritic area, referred here to as a "perisomatic" area. This implies that some signal is conveyed from the soma to the axon terminal. This signal cannot be conveyed by action potentials because the neurons at this age do not fire them. As a putative mechanism underlying this long-range "remote" control, we found a new form of calcium dynamics, which is initiated at the perisomatic region and propagates to the axon terminal over a distance of hundreds of micrometers. Premature neurons that are yet incapable of firing action potentials may use this propagating cytosolic signal as a long-range mediator.

\section{Materials and Methods}

Pharmacological agents. Glutamate, AMPA, NMDA, 6-cyano7-nitroquinoxoxaline-2,3-dione (CNQX), 1-naphthyl acetyl spermine (NASPM), ionomycin, and nifedipine were purchased from SigmaAldrich (St. Louis, MO). (1S,3R)-1-Aminocyclopentane-1,3dicarboxylic acid (ACPD) was from Tocris Cookson (Bristol, UK). Thapsigargin was from Nacalai Tesque (Kyoto, Japan). 2-Aminoethoxydiphenyl borate (2APB) was from Calbiochem (La Jolla, CA). Oregon Green 488 BAPTA-1AM was from Invitrogen (Carlsbad, CA). Sema3F was prepared as described previously (Yamada et al., 2005).

Time-lapse imaging from cultured granule cells and data analysis. Primary cultures of dentate granule cells and Ammon's horn neurons were prepared from postnatal day 3 Wistar/ST rats (SLC, Shizuoka, Japan), as described previously (Baba et al., 2002; Ichikawa et al., 2007). On day 4 in vitro, cells were mounted in a perfusion chamber (volume, $1.7 \mathrm{ml}$ ) put on transparent heating plate (MATS-505SF; TOKAI HIT, Shizuoka, Japan). To minimize solution convection caused by heating, medium was constantly perfused at a speed of $0.6 \mathrm{ml} / \mathrm{min}$ perpendicular to the axis from the soma to the axon terminal. This background flow was produced by adding medium to one edge of the chamber and removing it from the opposite side with a peristaltic pump. The medium, which consisted of 
98\% Neurobasal and 2\% B27 supplement (Invitrogen), was aerated with $95 \%$ air $/ 5 \% \mathrm{CO}_{2}$ and warmed at $32.5^{\circ} \mathrm{C}$ (in chamber). Phasecontrast images were acquired with a cooled CCD camera (Cascade 512B/F; Roper Scientific, Tucson, AZ) and a water-immersion objectives (40×; numerical aperture, 0.8; Achroplan; Carl Zeiss, Oberkochen, Germany), equipped with a Carl Zeiss AxioSkop2 upright microscope and custom-written image-acquisition controller based on MetaMorph (Molecular Devices, Union City, CA). Imaging was started 3 min before drug application and taken at $0.1 \mathrm{~Hz}$ for 8 min.

Neurons that touched other cells were not used. Axons were defined as the longest processes that were longer than $80 \mu \mathrm{m}$ and at least three times longer than all other neurites. Cells that did not possess such obvious neurites were not analyzed. All neurons selected with these criteria showed immunoreactivity for Prox-1, a marker of dentate granule cells (supplemental Fig. 1, available at www.jneurosci.org as supplemental material). Almost all (96.7\%) longest neurites were immunopositive for tau-1, an axon marker, whereas the other shorter processes were always tau-1-negative (supplemental Fig. 1, available at www.jneurosci.org as supplemental material). To measure axon length, $10-\mu \mathrm{m}$-radius circles were placed onto the entire axon so that the center of each circle was placed at the edges of the neighboring circles. Axon length was measured as the summed radii of this circle chain linking between the soma and the axon terminal. The length of each axon was normalized to the baseline at $0 \mathrm{~min}$ (i.e., immediately before the onset of drug application). Data were averaged across 5-13 cells.

Local drug application. Glass micropipettes (5-7 M $\Omega$ ) were pulled with a two-stage Narishige (Tokyo, Japan) puller and filled with drugs and Alexa 568 (1:1000), which were dissolved in perfusion medium. The tip of a micropipette was placed $30 \mu \mathrm{m}$ apart from the soma or axon terminal. The pipette was connected to a pressure-application system through $>2-\mathrm{m}$ long silicon tube (inner $\Phi=2.0 \mathrm{~mm}$ ). The other end of the tube was held $100 \mathrm{~cm}$ above the sample stage. The tube was filled with distilled water from this end to the position immediately before the microscopic stage, while on the stage, the tube was filled with air. To eject solution from the pipette, a positive pressure of $\sim 50 \mathrm{hPa}$ was applied with water gravity. Care was continuously taken to avoid a decline of air pressure as a function of time; to compensate this pressure decline, the top surface of the filled water was manually adjusted to be located immediately beneath the stage. In each experiment, Alexa 568 fluorescence was also monitored to estimate the drug concentration at the applied area. Alexa 568 was excited at $568 \mathrm{~nm}$ with an argon-krypton laser (Melles Griot, Carlsbad, CA) and imaged with a Cascade 512B/F camera and MetaMorph software.

Calcium imaging. On days 4 in vitro, cultures were transferred into a well of a 24-well plate filled with $1 \mathrm{ml}$ of Neurobasal medium containing $0.0005 \%$ Oregon Green 488 BAPTA-1AM and incubated for $1 \mathrm{~h}$ in a humidified incubator at $37^{\circ} \mathrm{C}$ in $5 \% \mathrm{CO}_{2}$. After being washed, cells were mounted in an imaging chamber perfused with $98 \%$ Neurobasal medium and 2\% B27 supplement. Images were taken at 2-5 frames/s with a Nipkow spinning-disk confocal microscope (CSU10; Yokogawa Electric, Tokyo, Japan), a Cascade 512B/F camera, and MetaMorph software. Fluorophores were excited at a $488 \mathrm{~nm}$ line with an argon-krypton laser current injection.

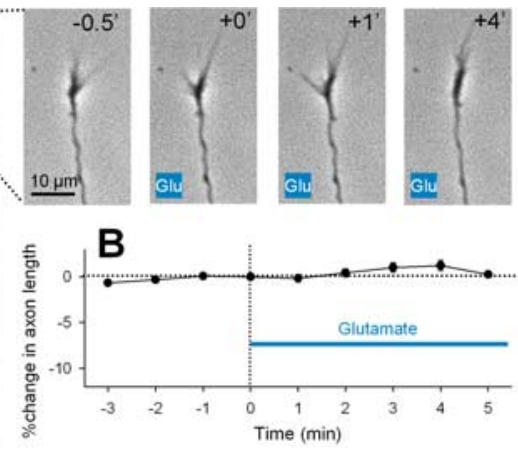

C Glutamate to axon tip

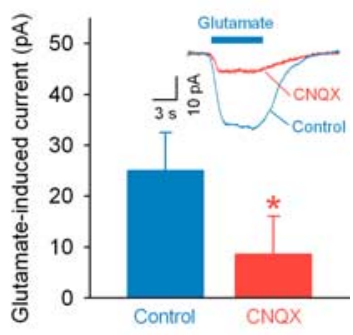

F Glutamate to perisoma

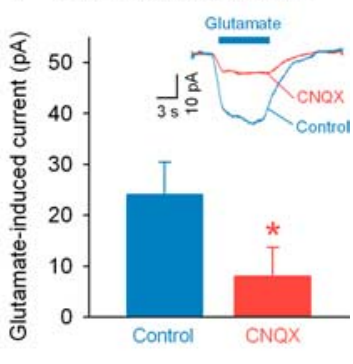

H

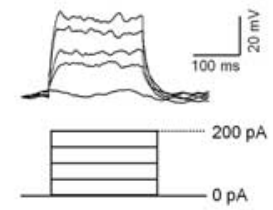

Figure 1. Local application of glutamate to the perisomatic region induces axon retraction. $\boldsymbol{A}$, Representative time-lapse images of the axon terminal in the inset box of the left whole-cell image. Numbers represent time (in minutes) after the start of application of glutamate ( $1 \mathrm{~mm}$ in pipette) to the axon terminal. $\boldsymbol{B}$, Glutamate-induced changes in axon length are summato the perisomatic region. The movie was taken from the same neuron as shown in the $A$. $E$, Data were summarized as means + application to the axon terminal $(\boldsymbol{C})$ and the perisoma $(\boldsymbol{F})$ induced a $C$ NQX-sensitive inward current. ${ }^{*} p<0.05$ versus control, Student's $t$ test; $n=4$. The insets indicate typical traces of glutamate-evoked currents in the presence and absence of CNQX at a glutamate application was stopped. $\boldsymbol{H}$, Immature granule neurons did not fire action potentials in responses to depolarizing

$(10 \mathrm{~mW})$ and visualized with a $507 \mathrm{~nm}$ long-pass emission filter. The fluorescence change $(\Delta F / F)$ was calculated as $\left(F_{t}-F_{0}\right) / F_{0}$, where $F_{t}$ is the fluorescence intensity in a $2-\mu \mathrm{m}$-radius circle placed on an axon at time $t$, and $F_{0}$ is the mean baseline value for a period of $20 \mathrm{~s}$ before drug application in the same circle.

Electrophysiologic recordings. For perforated patch-clamp recordings, $25 \mathrm{mg} / \mathrm{ml}$ gramicidin (Sigma-Aldrich; in DMSO) was diluted in $\mathrm{KCl}$ based intracellular solution composed of the following (in $\mathrm{mM}$ ): $130 \mathrm{KCl}$, $5 \mathrm{NaCl}, 0.4 \mathrm{CaCl}_{2}, 1 \mathrm{MgCl}_{2}, 1.1 \mathrm{EGTA}$, and 10 HEPES, pH 7.2 and 300 mOsm, immediately before experiments so that the final concentration was $50 \mu \mathrm{g} / \mathrm{ml}$. Recordings were performed with Axopatch 200B (Molecular Devices, Union City, CA). Signals were low-pass filtered at $1 \mathrm{kHz}$, digitized at $10 \mathrm{kHz}$, and analyzed with pCLAMP 8.0 software (Molecular Devices). Voltages were not corrected for liquid junction potential.

Immunostaining. Cultures were fixed for $20 \mathrm{~min}$ at $37^{\circ} \mathrm{C}$ with $4 \%$ paraformaldehyde, $10 \mu \mathrm{M}$ Taxol (Sigma-Aldrich), $1.3 \mu \mathrm{M}$ phalloidin (Sigma-Aldrich), $0.1 \%$ Triton-X, and $0.25 \%$ glutaraldehyde (Nacalai Tesque) in buffer consisting of $60 \mathrm{~mm}$ PIPES, $25 \mathrm{~mm}$ HEPES, $10 \mathrm{~mm}$ EGTA, and $2 \mathrm{mM} \mathrm{MgCl}_{2}$ at $\mathrm{pH}$ 6.9. They were incubated with $2 \%$ goat 

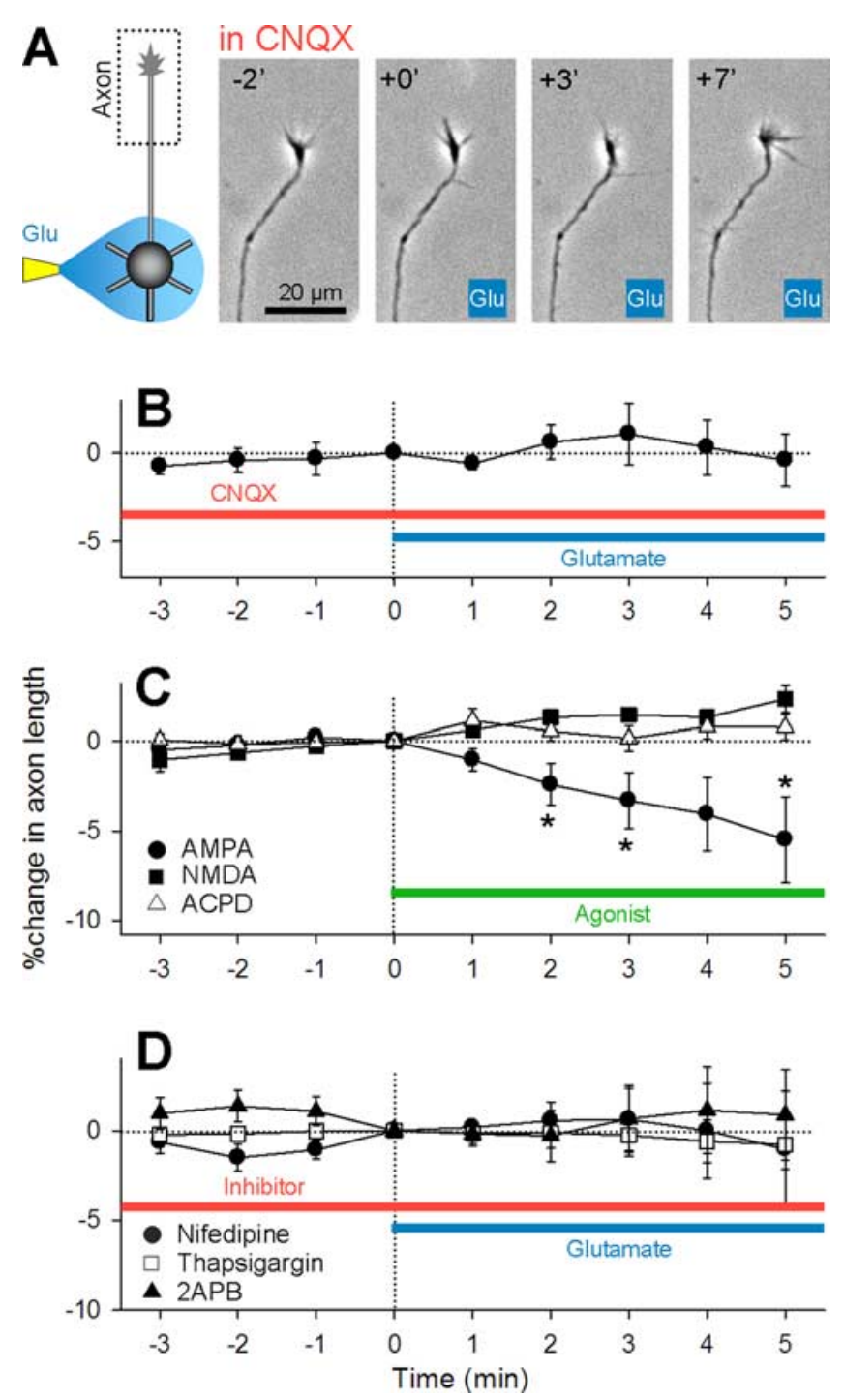

Figure 2. Glutamate-induced axon retraction requires non-NMDA receptor activity and intracellular calcium dynamics. $A$, Representative time-lapse images of the axon terminal of a neuron whose soma received glutamate application in the bath of $20 \mu \mathrm{m}$ CNQX. B, Summary data of perisomatic glutamate application in the presence of CNQX. Data represent means \pm SEM of eight axons. C, Local perisomatic application of AMPA (300 $\mu$ m in pipette) (circle) ( $n=$ 9), but not NMDA ( $1 \mathrm{~mm}$ in pipette) (square) ( $n=6)$ or ACPD ( $1 \mathrm{~mm}$ in pipette) (triangle) ( $n=$ 5), induced axon retraction. $\boldsymbol{D}$, Bath application of $10 \mu \mathrm{m}$ nifedipine (circle) $(n=7), 1 \mu \mathrm{m}$ thapsigargin (square) ( $n=12)$, and $100 \mu \mathrm{M} 2 \mathrm{APB}$ prevented the axon retraction (triangle) $(n=6) .{ }^{*} p<0.05$ versus 0 min, paired $t$ test.

serum in PBS for $1 \mathrm{~h}$ at room temperature, with the primary antibodies against rabbit anti-Prox1 (1:5000; AB5475; Millipore Bioscience Research Reagents, Temecula, CA) and mouse anti-tau-1 (1:2000; MAB3420; Millipore Bioscience Research Reagents) overnight at $4^{\circ} \mathrm{C}$, and then with Alexa 594-labeled anti-rabbit IgG (1:400; A-11037; Invitrogen) and Alexa 488-labeled anti-mouse IgG (1:400; A-11001; Invitrogen) in the presence of rhodamine-conjugated phalloidin (1:40; $\mathrm{R}-415$; Invitrogen) for $3 \mathrm{~h}$ at room temperature. Images were acquired with an ORCAII cooled CCD camera (Hamamatsu Photonics, Hamamatsu, Japan) and an ECLIPSE TE300 microscope (Nikon, Tokyo, Japan) with a $40 \times$ objective.

\section{Results}

Remote control of axon outgrowth

When primary cultures of granule cells were bathed in $500 \mu \mathrm{M}$ glutamate for $5 \mathrm{~min}$, growth cones collapsed in both axons and dendrites (supplemental Fig. 1, available at www.jneurosci.org as supplemental material), as we previously demonstrated (Yamada et al., 2005). To investigate the local effect of glutamate on growth cones, we developed an experimental system for local application of drugs to a small subregion of a cell (supplemental Fig. 2A, available at www.jneurosci.org as supplemental material). A glass pipette was located $30 \mu \mathrm{m}$ apart from the cell, and drugs were pressure-ejected with Alexa Fluor 568 (supplemental Fig. 2B, available at www.jneurosci.org as supplemental material). Because the Alexa fluorescence intensity was almost proportional to the Alexa concentration, we could estimate the spatial pattern of an Alexa diffusion gradient (supplemental Fig. $2 C$, available at www.jneurosci.org as supplemental material). The gradient was made within $3 \mathrm{~s}$ and kept stable at least for tens of minutes during continuous pressure. In each experiment, we quantified the Alexa fluorescence to approximate the drug concentration at the region of interest.

Glutamate applied to the axon terminal failed to induce any change in the axon behavior (Fig. $1 A, B$ ). Glutamate applied to the perisomatic region, however, readily led to a backward movement of axon terminals, resulting in a shortening of the total axon length (Fig. 1D,E). The degree of the axon retraction was positively correlated to the putative glutamate concentration at the perisomatic region (supplemental Fig. $3 A$, available at www. jneurosci.org as supplemental material), but uncorrelated with axon length before glutamate application (supplemental Fig. $3 B$, available at www.jneurosci.org as supplemental material). The remote effect of glutamate was not observed in putative pyramidal neurons prepared from hippocampal Ammon's horn $(n=5)$ (data not shown). Local application of sema3F, a potent growth cone repellent (Yamada et al., 2006), to the perisomata of granule cells induced axon retraction $(n=2)$ (data not shown).

Why was direct glutamate application on the axon ineffective in granule cells? As application of sema3F to axon terminals induced repulsive turning responses and facilitated axon elongation (supplemental Fig. 4, available at www.jneurosci.org as supplemental material), axonal growth cones themselves were responsive. Thus, one possibility is that glutamate receptors are absent in axon terminals. Whole-cell patch-clamp recordings revealed, however, that voltage-clamped granule cells showed an inward current in response to glutamate locally applied to the axon terminals (Fig. 1C). The current size was almost the same as that observed when glutamate was applied to the perisomatic area (Fig. $1 F$ ), and was significantly attenuated by bath application of CNQX, a non-NMDA ionotropic receptor antagonist (Fig. $1 C, F)$. Thus, axon terminals as well as perisomatic regions express functional glutamate receptors. We also rule out the possibility that glutamate-induced excitotoxicity caused axon shrinkage damage, because retracted axons began to reelongate soon after glutamate application was stopped (Fig. 1G).

We next addressed the mechanisms underlying glutamateinduced axon retraction pharmacologically. In the presence of CNQX, glutamate failed to induce axon retraction (Fig. 2A,B). Local application of AMPA, a non-NMDA receptor agonist, to perisomatic regions induced axon retraction (Fig. 2C), whereas either NMDA or the type I/II metabotropic receptor agonist ACPD did not (Fig. 2C). Thus, non-NMDA receptors mediate the action of glutamate, and their activation is sufficient to mimic the action of glutamate.

Because activation of these receptors leads to membrane potential depolarization, we next addressed the possible involvement of voltage-sensitive calcium channels. Bath application of nifedipine, an L-type calcium channel blocker, completely inhib- 
ited glutamate-induced axon retraction (Fig. 2D). Moreover, disturbance of intracellular calcium dynamics with thapsigargin, an inhibitor of $\mathrm{Ca}^{2+}$-ATPase of the endoplasmic reticulum, and $2 \mathrm{APB}$, an $\mathrm{IP}_{3}$ receptor inhibitor, inhibited axon retraction. Thus, depolarization and calcium signaling are required for glutamate-induced axon retraction.

\section{Axonal calcium sweep}

What signal propagates from the perisomatic region to the axon terminal and induces axon retraction? A plausible candidate is action potential. However, immature granule cells at day 4 in vitro were still incapable of generating action potential even when they received strong current injection (Fig. $1 H)(n=11)$. Another intriguing candidate is intracellular calcium wave (Jaffe, 1993; Berridge, 2002). To investigate this possibility, granule cells were loaded with Oregon Green 488 BAPTA-1AM and imaged with a Nipkowtype confocal microscope. After perisomatic application of glutamate, neurons exhibited a transient calcium increase in the entire cell, followed by a calcium increase that propagated from the perisoma to axon terminal (Fig. $3 A, B$; supplemental movie 1, available at www.jneurosci.org as supplemental material). We termed the latter unique dynamics "axonal calcium sweep." Local application of glutamate to axon terminal induced a fast calcium transient, but not axonal calcium sweep (Fig. $3 C$ ). Data are summarized in Figure $3 D$.

The velocity of the calcium sweep was on average $2.1 \pm 0.6 \mu \mathrm{m} / \mathrm{s}$, ranging from 0.9 to $3.2 \mu \mathrm{m} / \mathrm{s}$ (mean $\pm \mathrm{SD}$ of $n=21$ cells). Under control conditions, once the sweep was initiated, it did not disappear or slow down in the middle of the axon. Importantly, the arrival of axon calcium sweep at the axon tip always preceded axon retraction (supplemental movie 2, available at www.jneurosci.org as supplemental material). The time delay from the sweep arrival to the beginning of axon retraction was $57 \pm 21 \mathrm{~s}$ (mean $\pm \mathrm{SD}$ of $n=21$ cells), ranging from 12 to $89 \mathrm{~s}$. CNQX and nifedipine blocked both the glutamate-induced calcium transient and the sweep (Fig. $3 E, F$ ). In the presence of thapsigargin, the initial calcium increase was prolonged, and the second calcium sweep did not occur (Fig. 3G). In the presence of $2 \mathrm{APB}$, only the initial calcium transient occurred in five of six neurons (Fig. $3 H$ ), whereas one neuron exhibited "retrograde" calcium sweep from the axon tip to the soma $248 \mathrm{~s}$ after glutamate application.

To examine whether an increase in intracellular calcium is enough to evoke axon retraction, the calcium ionophore ionomycin was applied to the perisomatic region or axon terminal. Ionomycin evoked a prolonged calcium increase in the directly applied, small area, but it did not either elicit axonal calcium sweep or induce axon retraction (Fig. $4 A, B$ ). This suggests that a local calcium increase in the perisoma or axon terminal alone was insufficient to induce a global calcium event. Because glutamate induced a long-lasting inward current of $\sim 25 \mathrm{pA}$ (Fig. $1 F$ ), we used the patch-clamp recording technique to inject a DC current that mimicked the effect of glutamate. Injection of a $25 \mathrm{pA}$ current into current-clamped neurons for $300 \mathrm{~s}$ evoked a depolarization of $>10 \mathrm{mV}$ (data not shown), but it did not induce a calcium rise or axon retraction (Fig. 4C). However, when the same current was injected simultaneously with perisomatic ionomycin application, it readily induced axonal calcium sweep and axon retraction (Fig. $4 D$ ). Thus, the initiation of axonal calcium sweep requires a combination of depolarization and a perisomatic calcium increase.

Based on subunit compositions, non-NMDA glutamate receptors are classified into calcium-permeable and calciumimpermeable types (Hollmann et al., 1991; Verdoorn et al., 1991). That a combination of depolarization and calcium mimicked the glutamate effect suggests the involvement of the calcium-permeable type. We tested the effect of NASPM, an antagonist of calcium-permeable non-NMDA receptors (Blaschke 

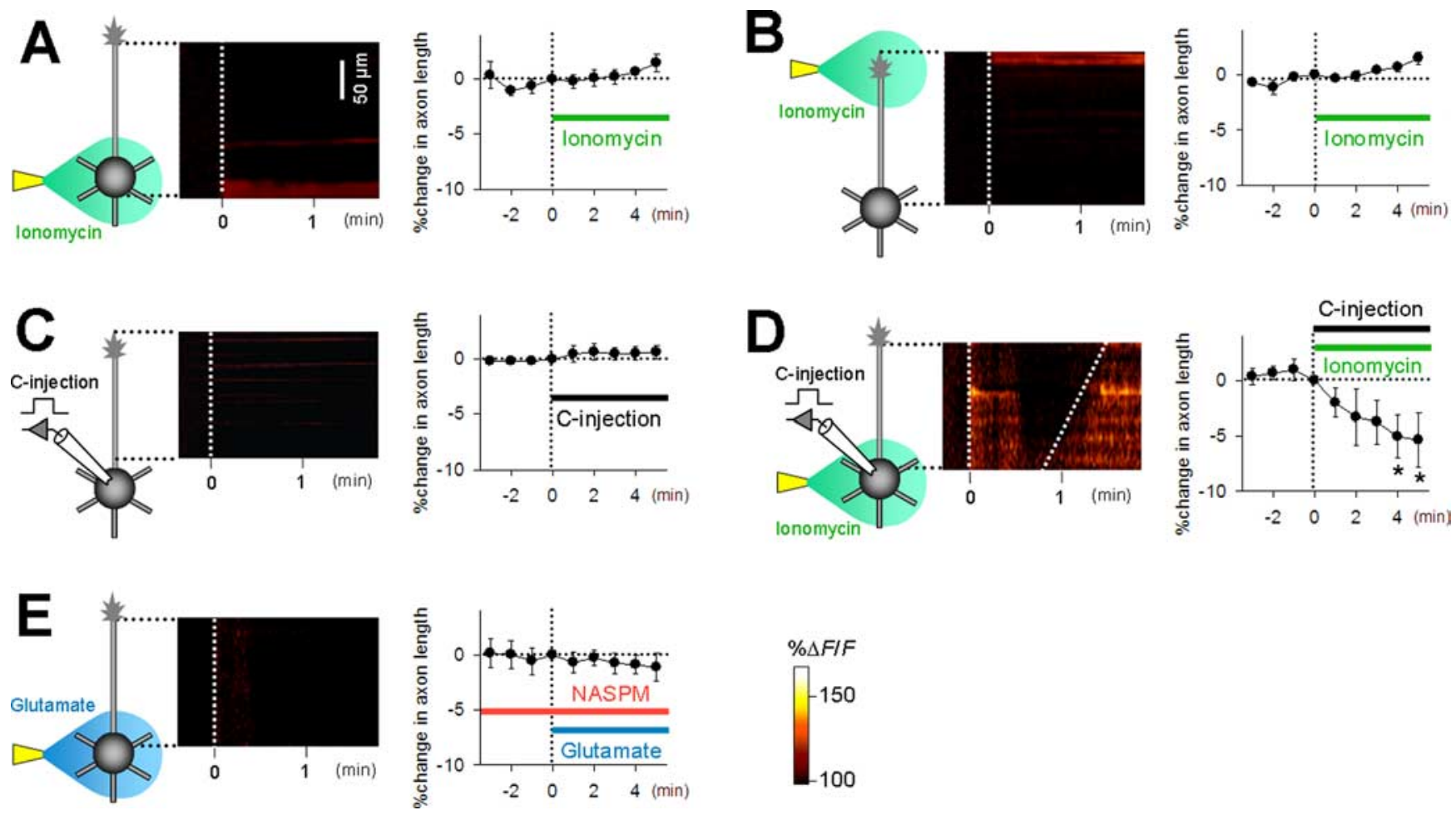

Figure 4. A combination of a somatic calcium rise and membrane depolarization induces axonal calcium sweep and axon retraction. $\boldsymbol{A}$, Local application of ionomycin (10 $\mu$ m in pipette) to the perisoma induced a calcium increase in the soma, but not the axonal calcium sweep (left), nor did it induce axon retraction (right) ( $n=6$ ). $\boldsymbol{B}$, Local application of ionomycin to the axon terminal induced a calcium increase in the axon tip, which did not lead to axon retraction $(n=5)$. $C$, Depolarization induced by current injection with the patch-clamp technique did not induce either axonal calcium sweep or axon retraction $(n=4)$. D, A combination of perisomatic ionomycin and current injection induced axonal calcium sweep and axon retraction. ${ }^{*} p<0.05$ versus 0 min, paired $t$ test $(n=6)$. $\boldsymbol{E}$, Bath application of $10 \mu \mathrm{m}$ NASPM inhibited both axonal calcium sweep and axon retraction induced by perisomatic glutamate application ( $n=6)$. Error bars indicate SEM.

et al., 1993). Bath application of NASPM inhibited glutamateinduced calcium sweep and axon retraction (Fig. $4 E$ ).

\section{Discussion}

In Xenopus spinal neurons and retinal neurons, growth cones display normal chemotaxic behavior even after being isolated from the parent cell bodies (Campbell and Holt, 2001; Ming et al., 2002). This can be interpreted as indicating that axonal growth cones respond specifically to their surrounding environment. We found, however, that the axons are also responsive to perisomatic glutamate, suggesting that the milieu surrounding the cell body affects the axon behavior.

\section{Roles of glutamate during brain development}

Most excitatory neurons, including dentate granule cells, use glutamate as a neurotransmitter. During development, glutamate modulates the formation of filopodia and the direction of neurite outgrowth (Lipton and Kater, 1989; Zheng et al., 1996; Koyama et al., 2002) and promotes a transition from filopodia to mature synapses (Fischer et al., 2000; Chang and De Camilli, 2001; Tashiro et al., 2003). Glutamate is also involved in the selective survival and network formation of immature granule cells (Tashiro et al., 2006). Therefore, our findings that glutamate alters the neurite behaviors were not surprising. But we underline that immature granule cells that had not encountered other neurons already expressed functional glutamate receptors, even in axons, and that these ionotropic glutamate receptors exerted a distant effect on axon terminals far from where the receptors were activated. In general, calcium through glutamate receptor channels acts locally, in particular, to enhance the input specificity of synaptic plasticity. The biological function of perisomatic glutamate-induced axon retraction remains to be elucidated, but we speculate that in vivo glutamate spills over from synaptic clefts of neighboring neurons. Because hippocampal pyramidal cells did not respond to perisomatic glutamate, dentate granule cells may be specifically endowed with the ability of perisomatic axon control. Given that granule cells continue to be produced even in the adult brain (Lledo et al., 2006), activation of dentate gyrus synapses (e.g., mature perforant-path synapses) could retard or halt the axon extension of nearby immature granule cells and change the targeting destination of their premature axons.

\section{Unique calcium dynamics in axon}

Past studies on axonal calcium dynamics have focused mainly on presynaptic terminals or growth cones, and the calcium dynamics in axon stalks is ill defined. Guan et al. (2007) have recently reported that the leading processes of migrating cerebellar granule neurons generate calcium waves that propagate from the growth cone to the soma to cause reversal of cell polarity and migration. This is consistent with our findings that the soma and axon can interact through long-range calcium dynamics within neurons. Because their and our calcium waves are both whole-cell phenomena coordinated by voltage-dependent calcium channels and intracellular calcium store, they may share common mechanisms. It is noteworthy that, unlike the leading processes of migrating neurons, the axons of developing granule cells are extremely narrow. Technically, we achieved calcium imaging from axons with Nipkow-type confocal microscopy, which is designed to minimize photodamage and photobleaching (Wang et al., 2005; Sasaki et al., 2007).

We found that axonal calcium sweeps propagate at a speed of $\sim 2 \mu \mathrm{m} / \mathrm{s}$. This velocity is a few times slower than generally 
known $\mathrm{IP}_{3}$-dependent regenerative calcium wave (Jaffe, 1993; Berridge, 2002). $\mathrm{IP}_{3}$ waves are known to be attenuated by cGMP signaling pathway (Sergeant et al., 2006). Because our previous study showed that cGMP is involved in axon growth of dentate granule cells (Yamada et al., 2006), it is possible that interacting $\mathrm{IP}_{3}$ and cGMP generates an unusually slow calcium wave.

Although our study does not strictly indicate whether axonal calcium sweep mediates axon retraction, three observations suggest a causal link: (1) calcium sweep always preceded axon retraction; (2) pharmacological sensitivity was similar between calcium sweep and axon retraction, that is, all experimental manipulation that inhibited the generation of calcium sweep inhibited axon retraction; (3) experimental manipulation that initiated calcium sweep induced axon retraction. However, it should be noted that the arrival of a calcium sweep at the axon terminal is unlikely by itself to trigger axon retraction, because experiments with calcium ionophore indicate that a local calcium elevation in the axon terminal was insufficient to induce axon retraction, and also because the calcium increase prolonged by thapsigargin did not induce axon retraction. The crucial aspect of calcium sweep is, hence, not the arrival of a "calcium spot" at the axon tip, but rather its spatiotemporal calcium dynamics throughout the cell. More specifically, we speculate that biphasic calcium increases may serve as priming and trigger; for example, the initial calcium transient might induce phosphorylation of microtubule-associated protein $1 \mathrm{~b}$ and tau through calcium/ calmodulin-dependent kinase and destabilizes microtubules (priming), and then, the second calcium wave causes microtubule depolymerization (trigger). Investigating the molecular mechanisms is underway in our laboratory.

\section{References}

Baba A, Yamada MK, Nishiyama N, Matsuki N, Ikegaya Y (2002) Different $\mathrm{Ca}^{2+}$ dynamics between isolated hippocampal pyramidal cells and dentate granule cells. J Neurocytol 31:41-48.

Berridge MJ (2002) The endoplasmic reticulum: a multifunctional signaling organelle. Cell Calcium 32:235-349.

Blaschke M, Keller BU, Rivosecchi R, Hollmann M, Heinemann S, Konnerth A (1993) A single amino acid determines the subunit-specific spider toxin block of alpha-amino-3-hydroxy-5-methylisoxazole-4-propionate/ kainate receptor channels. Proc Natl Acad Sci USA 90:6528-6532.

Campbell DS, Holt CE (2001) Chemotropic responses of retinal growth cones mediated by rapid local protein synthesis and degradation. Neuron 32:1013-1026.

Chang S, De Camilli P (2001) Glutamate regulates actin-based motility in axonal filopodia. Nat Neurosci 4:787-793.

Fischer M, Kaech S, Wagner U, Brinkhaus H, Matus A (2000) Glutamate receptors regulate actin-based plasticity in dendritic spines. Nat Neurosci 3:887-894.

Guan CB, Xu HT, Jin M, Yuan XB, Poo MM (2007) Long-range $\mathrm{Ca}^{2+}$ sig- naling from growth cone to soma mediates reversal of neuronal migration induced by slit-2. Cell 129:385-395.

Hollmann M, Hartley M, Heinemann S (1991) $\mathrm{Ca}^{2+}$ permeability of KAAMPA-gated glutamate receptor channels depends on subunit composition. Science 252:851-853.

Huber AB, Kolodkin AL, Ginty DD, Cloutier JF (2003) Signaling at the growth cone: ligand-receptor complexes and the control of axon growth and guidance. Annu Rev Neurosci 26:509-563.

Ichikawa J, Yamada RX, Muramatsu R, Ikegaya Y, Matsuki N, Koyama R (2007) Cryopreservation of granule cells from the postnatal rat hippocampus. J Pharmacol Sci 104:387-391.

Jaffe LF (1993) Classes and mechanisms of calcium waves. Cell Calcium 14:736-745.

Koyama R, Yamada MK, Nishiyama N, Matsuki N, Ikegaya Y (2002) Group II metabotropic glutamate receptor activation is required for normal hippocampal mossy fibre development in the rat. J Physiol (Lond) 539:157-162.

Lipton SA, Kater SB (1989) Neurotransmitter regulation of neuronal outgrowth, plasticity and survival. Trends Neurosci 12:265-270.

Lledo PM, Alonso M, Grubb MS (2006) Adult neurogenesis and functional plasticity in neuronal circuits. Nat Rev Neurosci 7:179-193.

Ming GL, Wong ST, Henley J, Yuan XB, Song HJ, Spitzer NC, Poo MM (2002) Adaptation in the chemotactic guidance of nerve growth cones. Nature 417:411-418.

Sasaki T, Matsuki N, Ikegaya Y (2007) Metastability of active CA3 networks. J Neurosci 27:517-528.

Sergeant GP, Johnston L, McHale NG, Thornbury KD, Hollywood MA (2006) cGMP/PKG pathway inhibits electrical activity in rabbit urethral interstitial cells of Cajal by reducing the spatial spread of $\mathrm{Ca}^{2+}$ waves. J Physiol (Lond) 574:167-181.

Tashiro A, Dunaevsky A, Blazeski R, Mason CA, Yuste R (2003) Bidirectional regulation of hippocampal mossy fiber filopodial motility by kainate receptors: a two-step model of synaptogenesis. Neuron 38:773-784.

Tashiro A, Sandler VM, Toni N, Zhao C, Gage FH (2006) NMDA-receptormediated, cell-specific integration of new neurons in adult dentate gyrus. Nature 442:929-933.

Tessier-Lavigne M, Goodman CS (1996) The molecular biology of axon guidance. Science 274:1123-1133.

Verdoorn TA, Burnashev N, Monyer H, Seeburg PH, Sakmann B (1991) Structural determinants of ion flow through recombinant glutamate receptor channels. Science 252:1715-1718.

Wang E, Babbey CM, Dunn KW (2005) Performance comparison between the high-speed Yokogawa spinning disc confocal system and single-point scanning confocal systems. J Microsc 218:148-159.

Yamada RX, Matsuki N, Ikegaya Y (2005) cAMP differentially regulates axonal and dendritic development of dentate granule cells. J Biol Chem 280:38020-38028.

Yamada RX, Matsuki N, Ikegaya Y (2006) Soluble guanylyl cyclase inhibitor prevents Sema3F-induced collapse of axonal and dendritic growth cones of dentate granule cells. Biol Pharm Bull 29:796-798.

Zheng JQ, Wan JJ, Poo MM (1996) Essential role of filopodia in chemotropic turning of nerve growth cone induced by a glutamate gradient. J Neurosci 16:1140-1149. 